\title{
Minimal Invasive Transforaminal Lumbar Interbody Fusion for Isthmic Spondylolisthesis with Nerve Root Anomaly in Young Adult Male Football Player: Case Report
}

\author{
Umesh Srikantha ${ }^{1}$, Yadhu K Lokanath ${ }^{1}$, Akshay Hari ${ }^{1}$, Ravi Gopal Varma ${ }^{1}$
}

\section{Abstract}

Interbody fusion is preferred surgical treatment for various symptomatic pathologies of lumbar spine and spondylolisthesis is one of the commonest indication. Goal is to achieve adequate bony and neural decompression, disc height restoration, deformity correction and good interbody fusion. Numerous techniques and approaches have been designed, minimal invasive tubular transforminal interbody fusion is widely accepted among them. In any case pre-operative planning and identifying any anatomical variation is prerequisite before surgery. In this article, we report, 22-year male football player, presenting with worsening low back pain, left radicular pain with neurogenic claudication. Radiographs revealed a L5-S1 Meyerding grade 1 Isthmic spondylolisthesis with L5-S1 left sub articular disc protrusion with narrowing of lateral recess on left side and no evidence of lumbosacral nerve root anomalies on magnetic resonance imaging. Patient was planned for minimal invasive L5-S1 transforaminal interbody fusion, intraoperatively, Type 3 Neidre and Macnab root anomaly was identified, patient successfully underwent interbody fusion with no acute intraoperative or postoperative complications. In follow up period, patient had no radicular pain. In our article, we summarize the incidence, classification of nerve root anomaly along with intra operative strategy for interbody fusion in presence of root anomaly and report this rare twin anomaly.

Keywords: Isthmic spondylolisthesis; Nerve root anomaly; MIS TLIF; Young football player.

\section{Introduction}

Spondylolysis and spondylolisthesis are the most common causes of low back pain. Reported incidence of isthmic spondylolisthesis (IS) is around $4 \%$ to $8 \%$ in general population [1].

Surprisingly in football players most common cause of low back pain is also spondylolysis with a reported incidence of 14 $\%$ [2]. IS results due to repetitive recurrent mechanical injury to pars resulting in subluxation over a period of time. IS is commonly seen in people who are involved in contact sports like football, hockey, rugby and other sports like gymnastics, dance, and figure skating which requires repetitive flexion, extension and torsion [3]. Pars defect and listhesis are the pain generators in these cases affecting their daily and routine activities.

Spinal nerves root anomalies are well described in literature

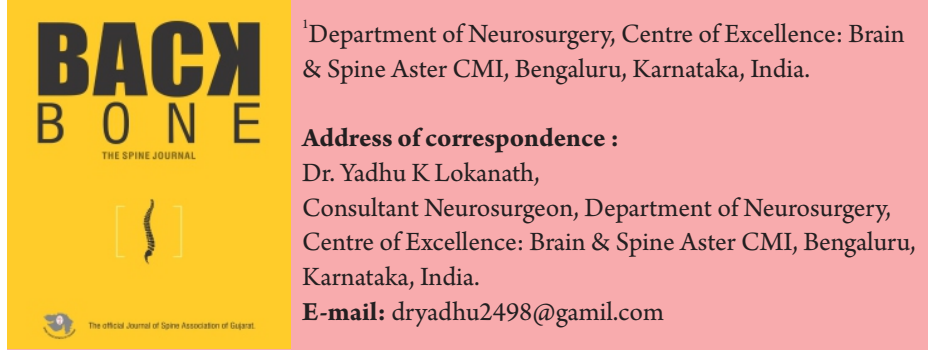

and are identified intraoperatively in majority of cases and not diagnosed preoperatively. From surgical point of view, combination of listhesis and nerve root anomaly requiring interbody fusion becomes technically demanding procedure as discectomy is difficult [4] to perform as roots are tethered, difficult to retract dural sac and roots consequently susceptible to injury while retraction maneuverer $[4,5]$ during cage insertion. Literature review shows rarity of reports of IS associated lumbosacral nerve root anomaly in young adolescent soccer players. Herein, we report a case of grade I isthmic spondylolisthesis in a young adolescent soccer player with persistent back and radicular pain who underwent minimal invasive (MI) interbody fusion and was symptom free during the follow up period. Given the rarity of this type of dual anomaly we report this case, describe the intraoperative strategy to deal these types of cases which do not permit easy access to disc space and classify the root anomalies with a focus on preoperative imaging to identify nerve root anomaly.

\section{Case Report}

22-year-old young football player presenting with history of low back pain more than year which has gradually progressed and worsened in the last 6 months. Over the last few months patient reports left lower limb radicular pain syndrome with

2021 (C) Back Bone: The Spine Journal (The Official Journal Of "Spine Association Of Gujarat") | Available on www.backbonejournal.com| DOI:10.13107/bbj.2021.v02i01.018 This is an Open Access article distributed under the terms of the Creative Commons Attribution Non-Commercial License (http://creativecommons.org/licenses/by-nc/3.0) which permits unrestricted non-commercial use, distribution, and reproduction in any medium, provided the original work is properly cited. 


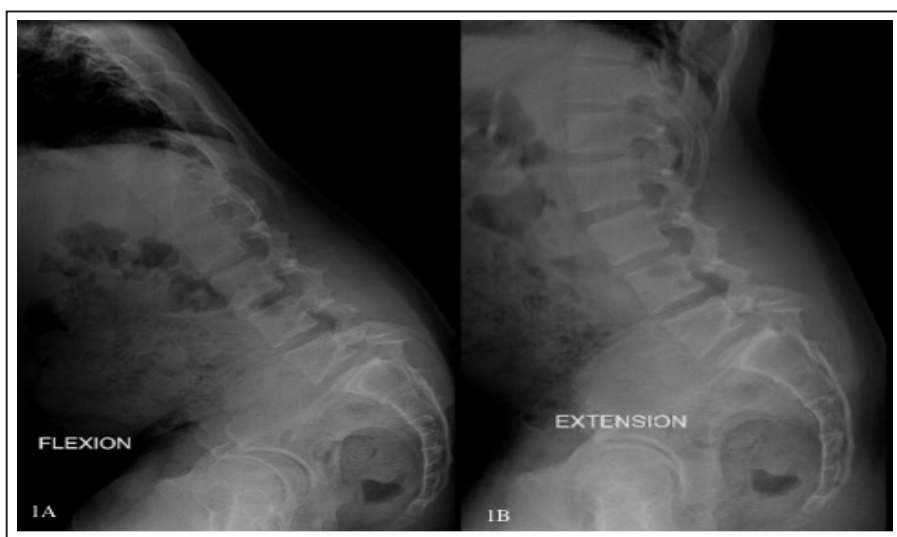

Figure 1: (A and B): Dynamic $\mathrm{X}$-rays of lumbosacral spine showing L5-S1 grade 1 spondylolisthesis with pars defect

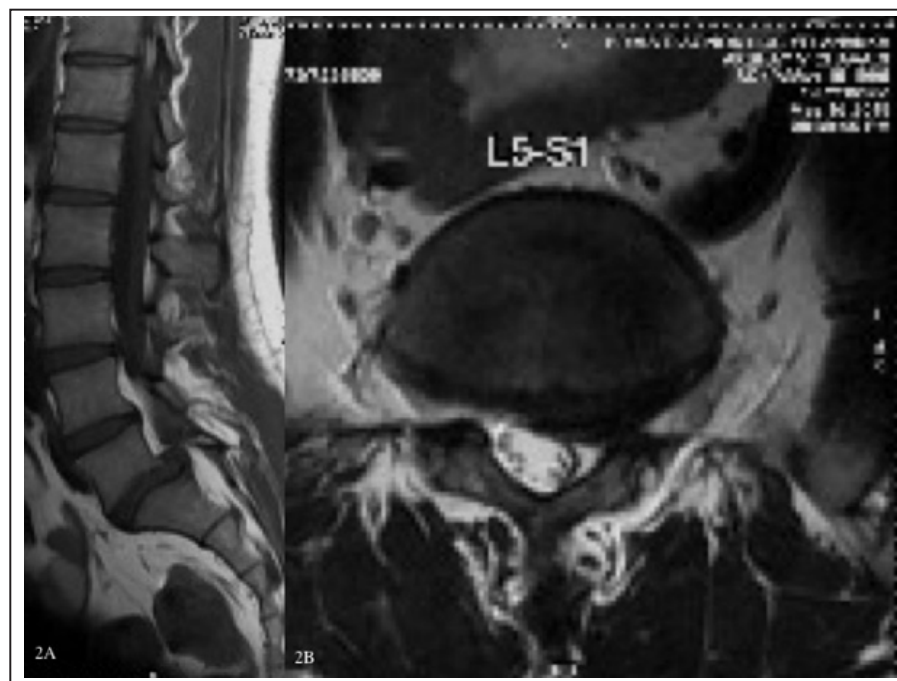

Figure 2: $(\mathrm{A}$ and $\mathrm{B})$ : Magnetic resonance imaging - Sagittal and axial T2 sequences showing L5-S1 left sub articular disc protrusion (pseudo disc bulge ) with narrowing of left lateral recess with grade I spondylolisthesis

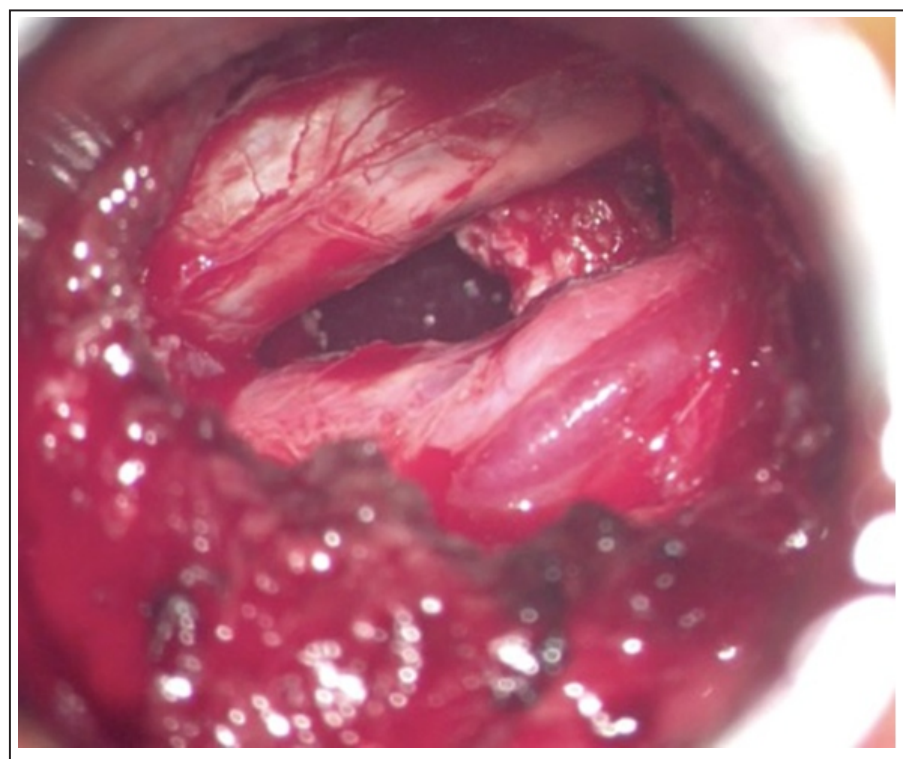

Figure 3: Intraoperative image showing anomalous $S 1$ nerve root was noted after facetectomy probably an anosmatic Type 3 Neidre and Macnab root anomaly (Tubular view) neurogenic claudication. Conservative measures by oral and intravenous analgesics provided no long-lasting benefits. Patients neurology was intact with positive straight leg raise test only up to $10^{\circ} \mathrm{on} \mathrm{left} \mathrm{side.} \mathrm{Sensation} \mathrm{were} \mathrm{intact} \mathrm{and} \mathrm{normal}$ deep tendon reflexes. Routine standing lumbosacral dynamic $x$ rays revealed L5-S1 grade 1 spondylolisthesis with pars defect (Fig. 1A \& B). Magnetic resonance imaging revealed L5-S1 left sub articular disc protrusion with narrowing of left lateral recess, but did not detect any lumbosacral nerve root anomaly (Fig. 2). Based on observation of patient symptoms and imaging, the need of surgery was the probable solution. Goal was to decompress the nerve root and interbody fusion with reduction of listhesis. MI transforaminal interbody fusion with L5-S1 percutaneous pedicle screw fixation was planned. Patient was placed prone under general anaesthesia. Mild reduction of listhesis was achieved after prone positioning, desired levels were marked and confirmed under fluoroscopy. We routinely use intraoperative navigation in our setup. Reference frame for navigation was placed on right posterior superior iliac spine. Pedicle entry points were marked on both side under navigation guidance. On right side L5 and S1percutaneous pedicle screw fixation was performed using $6.5 \times 45 \mathrm{~mm}$ polyaxial screws. On left side (symptomatic side) access to L5-S1 disc space was gained using $22 \mathrm{~mm} \times 5 \mathrm{~cm}$ METRx system (Medtronic Sofamor Danek, Memphis, TN). After initial docking, soft tissue dissection was performed to expose L5-S1 facet. Inferior facet was unstable with facet hypertrophy. Inferior facet of L5 with ascending part of superior facet of $\mathrm{S} 1$ was resected to accomplish facetectomy and access to disc space. Intraoperatively anomalous $\mathrm{S} 1$ nerve root was noted after facetectomy (Fig. 3) probably an Type 3 Neidre and Macnab root anomaly hindering access to disc space and inability to retract nerve root because bony confines and adhesions. Complete pedicle to pedicle decompression followed removal of ligamentum flavum and drilling of lamina was performed until lateral border of thecal sac was visible. At this point retraction of dural tube, nerve root was attempted, additional soft tissue removal was performed laterally, cranial and caudal with additional drilling of lamina medially enabling to retract the nerve root to expose disc space. Routine box annulotomy and discectomy was performed with end plates prepared for cage insertion. For intervertebral body fusion, we used $12 \mathrm{~mm}$ height and $24 \mathrm{~mm}$ length PEEK cages filled with autologous cancellous bone after insertion of trail cage on $\mathrm{X}$ ray. Interbody cage insertion was facilitated by using two nerve retractors for retraction of dural tube and traversing nerve root medially and anomalous nerve root laterally (Fig. 4). Left side L5 and S1 percutaneous pedicle screw fixation was done using $6.5 \times 45 \mathrm{~mm}$ screws and interconnected with $40 \mathrm{~mm}$ rods to achieve complete reduction of listhesis. Routine closure in layers after insertion suction drain was performed. 


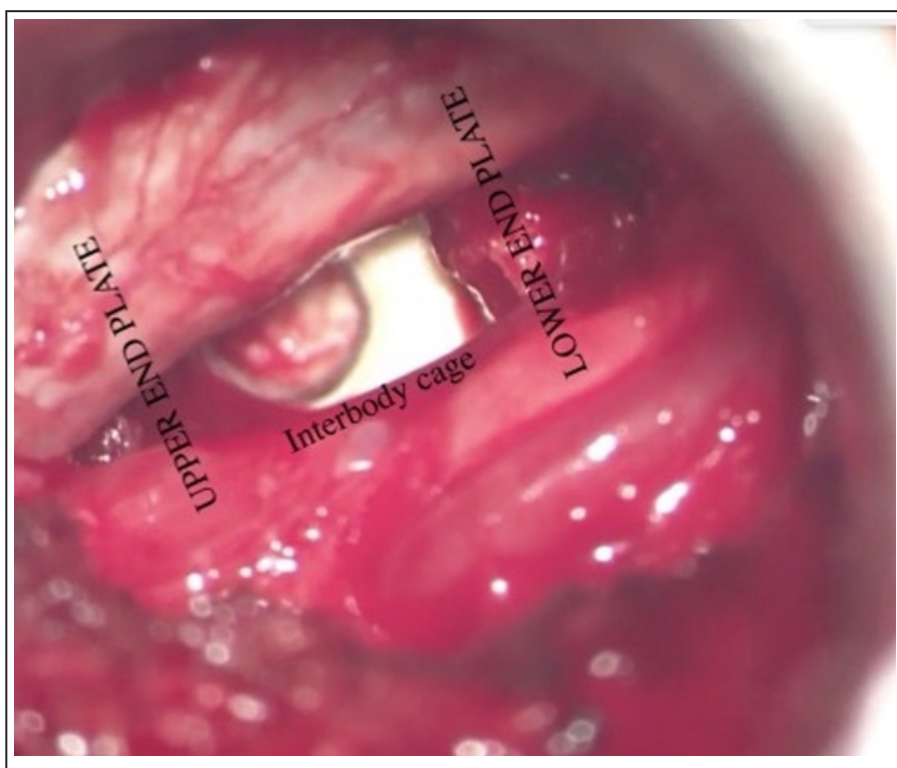

Figure 4: Intraoperative image showing interbody cage (White colour)

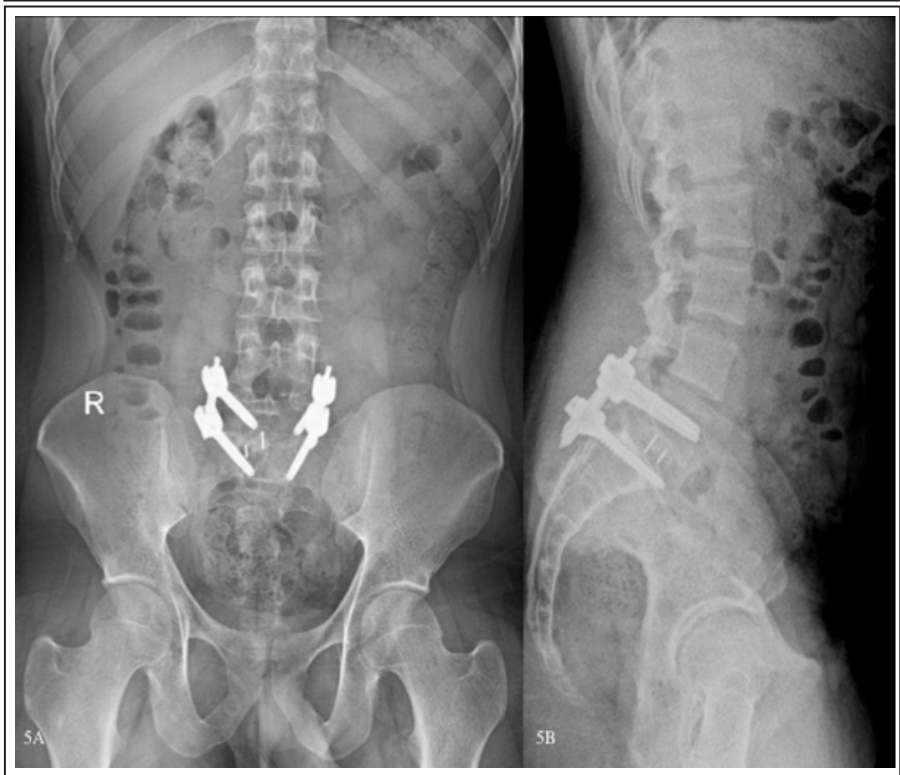

Figure 5: ( $\mathrm{A}$ and $\mathrm{B})$ : Post-operative Lumbosacral spine $\mathrm{x}$ ray (Anterio posterior / Lateral view) showing L5-S1 interbody cage with disc height restoration and reduction of spondylolisthesis
Postoperatively during follow up period, patient was relieved of his pre-operative symptoms and radiographs showed good alignment (Fig.5).

\section{Discussion}

Lumbosacral Nerve Root Anomalies (LNRA) are congenital anomalies developing due to aberrant migration of the involved roots during embryologic development and can be bifid, conjoined, transverse course or anastomosed nerve roots [1] with prevalence of $8.5-30 \%$ in autopsies [2]. In its normal course nerve root runs from medial to lateral direction along the inferior surface of the corresponding pedicle, exiting through the intervertebral foramen laterally [3]. The classification of LRNA is based on whether the nerve root emerges at an abnormal cranial or caudal level or from an anastomotic branch. The Neidre and Macnab classification is widely used and is classified accordingly.

Type 1 anomaly has conjoined nerve roots, $1 \mathrm{~A}$ two nerve roots arise from common dural sheath, $1 \mathrm{~B}$ nerve roots are conjoined producing a nerve root that exit at right angles to dural sheath in a manner of cervical root. In Type 2, two nerve roots exit from one foramen, $2 \mathrm{~A}$ this anomaly may leave one root canal unoccupied and in $2 \mathrm{~B}$ there may be nerve roots in all foramina but one foramen contains two separate nerve roots, Type 3, adjacent nerve roots are connected by a connecting in the form of an anastomosis and lastly Type 4 is combination of previously mentioned types with a combined types 2 and 3 anomalies [4] (Fig. 6).

In most cases the involved nerve roots are located at L5-S1 level with incidence of 52.5\%, while S1-S2 anomalies are observed in $30.4 \%$ [6]. LRNA are rarely diagnosed preoperatively and best imaging modality to identify is $\mathrm{T} 2$ coronal and T1 axial sequence. Song et al [7] described three radiological signs on standard axial MRI, "corner sign", "fat crescent sign" and "parallel sign" lastly Kang et al [8] described the "sagittal shoulder sign" on coronal sequence to identify the anomalous root. These signs are not conclusive but suggest the presence of anomalous root. Literature review reveals paucity

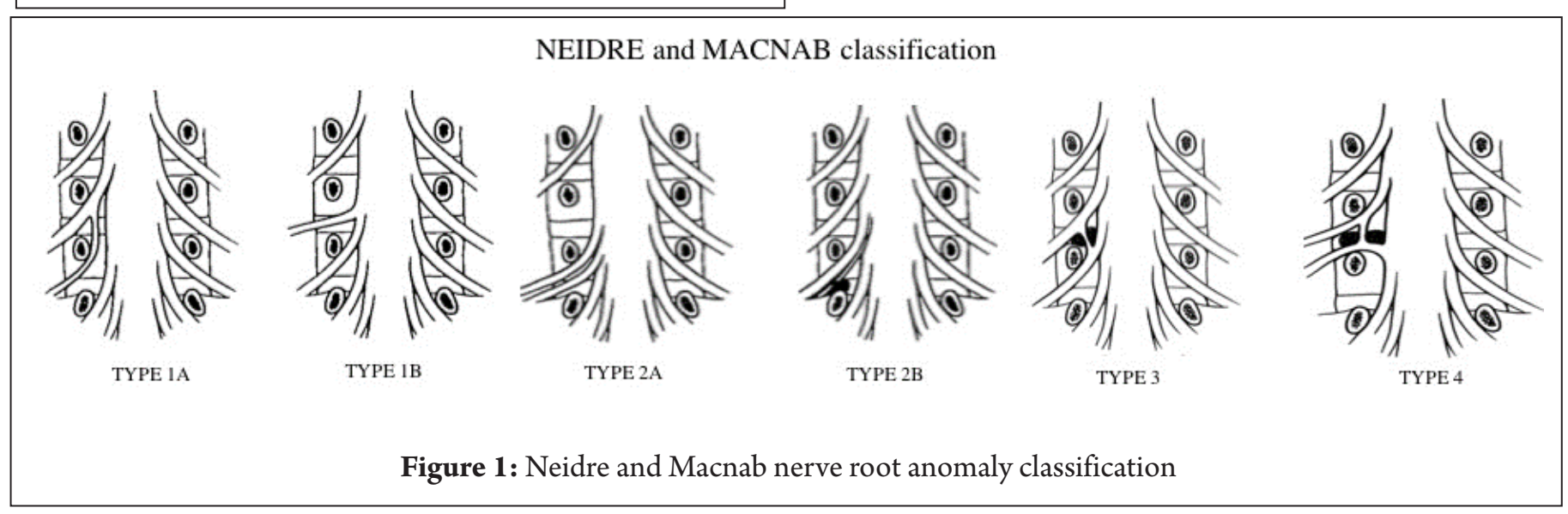

45 | Back Bone: The Spine Journal | Volume 2 | Issue 1 | April-September 2021 | Page 43-47 
of reports of lumbosacral anomalies in association with IS in young adolescents [9].

Spondylolisthesis are of many types and IS is one of the subtypes. Portion of neural arch that connects the posterior elements is referred as pars interarticularis, or isthmus. Pars is key components in maintaining segmental integrity. Defect in the pars interarticularis gradually progresses to vertebral body subluxation over a period of time and is referred as IS [10]. IS has multifactorial in origin. Repetitive trauma due to cyclical flexion-extension movements, axial and rotational loading leads to fatigue micro fractures of pars. These fractures sometimes heal or have fibrous union and have tendency to give away. Symptomatic IS warrants treatment in the form interbody fusion [11].

Interbody fusion can be from anterior, posterior or lateral approach in a conventional method or MI form which encompasses transformational lumbar interbody fusion (TLIF), anterior lumbar interbody fusion (ALIF), posterior lumbar interbody fusion (PLIF) and lateral lumbar interbody fusion (LLIF) which can be direct lateral lumbar interbody fusion (DLIF) or Oblique lumbar interbody fusion (OLIF). There are no clear definitive indications or evidences supporting superior to one another in terms of fusion or clinical outcomes. Each technique has its own indications with relation to patient's anatomy and pathological element. In this case MI TLIF procedure was planned, routinely which involves facet exposure, resection of facet, laminectomy, discectomy, end plate preparation, graft bed preparation, cage insertion and to conclude percutaneous pedicle screw fixation. In anatomical variation and pedicle screw in place on one side as in our case and anomalous root being identified on contralateral side requires case based alteration of surgical steps depending upon the intraoperative anatomy. In presence of LRNA because of anomalous nerve root, their size and attachment to surrounding structure, any attempt to retract or manipulate the nerve without adequate exposure and decompression leads to traction nerve injury. In this scenario, the other options to access the disc space are, from contralateral side or to approach through a lateral or anterior corridor. In situation where contralateral pedicle screws are already placed as in our case, access from ipsilateral side is inevitable. Presence of anomalous nerve root warrants wide exposure for access, adequate mobilisation and visualization to trace origin of nerve root, as described in our technique complete facetectomy, pedicle to pedicle decompression, lateral to medial decompression, contralateral laminotomy, unroofing of lateral recess and foraminotomy to have larger working zone lateral to thecal sac, exiting nerve root and caudal pedicle [12]. After complete exposure of osseous elements the ligamentum flavum(LF) is exposed. LF should be completed detached from its origin, insertion and towards midline. What precludes to look closely for a nerve root anomaly intraoperatively [12] are 1) an atypical location of a nerve root, in which the L-4 nerve root exited just rostral to the L-5 pedicle instead of just caudal to the L-4 pedicle (in case of L4-L5 pathology) 2) a nerve root that exits the thecal sac at an atypical angle 3 ) difficulty mobilizing a nerve root despite satisfactory pedicle-to-pedicle decompression. Discectomy and placing an adequate sized interbody graft to maintain disc height is also demanding technique in presence of anomalous nerve root. Discectomy in piece meal fashion with progressive retraction of roots, use interbody shavers and distraction devices to complete discectomy. Placing the interbody graft from contralateral is a viable option if needed, but as in our case we used two nerve root retractors for retraction and cage was inserted. Before inserting one must decide whether increasing the height of the intervertebral foramen is needed or placing an expandable device or pedicle screw distraction [9]. Correlation between clinical and imaging findings, understanding pathological anomalous anatomy, adhering to principles of decompression and meticulous handling of neural structures should be the principles for a successful outcome.

\section{Conclusion}

Interbody fusion is increasingly gaining popularity and is best surgical option in a symptomatic disease. Points taken into consideration are when a neurological finding exceeds more than anticipated more detailed assessment and thorough screening of imaging modalities should be performed. Detection of any anomaly preoperatively is the ideal way to plan the surgery. MI procedures can be performed in LRNA with adequate precaution and decompression has to carried out to avoid devastating results. 


\section{References}

1. Trimba R, Spivak JM, Bendo JA. Conjoined nerve roots of the lumbar spine. Spine J 2012;12:515-24.

2. Postacchini F, Urso S, Ferro L. Lumbosacral nerve-root anomalies. J Bone Joint Surg Am 1982;64(5):721-9.

3. Babayeva N, Torgutalp SS, Dönmez G Et al. Spondylolysis in an Adolescent Soccer Player.Austin Sports Med.2017; 2(1): 1013

4. Scuderi GJ, Vaccaro AR, Brusovanik GV et al. Conjoined lumbar nerve roots: a frequently underappreciated congenital abnormality. J Spinal Disord Tech 2004; 17:86-93.

5. Kadish LJ, Simmons EH. Anomalies of the lumbosacral nerve roots. An anatomical investigation and myelographicstudy. JBone Joint Surg Br 1984; 66:11-16.

6. Song SJ, Lee JW, Choi JY et al. Imaging features suggestive of a conjoined nerve root on routine axial MRI. Skeletal Radiol 2008;37:133-8.
7. Kang $C H$, Shin MJ, Kim SM et al. Conjoined lumbosacral nerve roots compromised by disk herniation: sagittal shoulder sign for the preoperative diagnosis. Skeletal Radiol 2008;37:225-31.

8. Davidson D,Rowan R, Reilly C : Lumbosacral Nerve Root Anomaly Associated With Spondylolisthesis in an Adolescent: A Case Report and Review of the Literature: SPINE Volume 31, Number 19,2006; pp E718 -E721

9. Hutton WC, Cyron BM, Spondylolysis. The role of the posterior elements in resisting the intervertebral compressive force. Acta Orthop Scand 1978; 49:604-609

10. Ganju A: Isthmic spondylolisthesis: Neurosurg Focus 2002 Jul 15;13(1):E1.

11. Moskowitz A: Transforaminal lumbar interbody fusion. Orthop Clin North Am 2002; 33:359-366

12. Shane MB, Mina GS, Kryzanski J et al : Nerve root anomalies: implications for transforaminal lumbar interbody fusion surgery and a review of the Neidre and Macnab classification system : Neurosurg 2013; Focus 35 (2):E9
Conflict of Interest: NIL Source of Support: NIL

\section{How to Cite this Article}

Srikantha S, Lokanath YK, Hari A, Varma RG | Minimal Invasive Transforaminal Lumbar Interbody Fusion for Isthmic Spondylolisthesis with Nerve Root Anomaly in Young Adult Male Football Player: Case Report | Back Bone: The Spine Journal | April-September 2021; 2(1):43-47. 